\title{
Photorefractive Grating in Multiple Quantum Well Planar Waveguide
}

\author{
E. WeinerT-RĄCZKA*, M. WiChtowski, A. ZıóŁKowski
}

AND G. STAROŃ

Institute of Electronics, Telecommunication and Informatics

Electrical Engineering Department, Technical University of Szczecin

al. Piastów 17, 70-310 Szczecin, Poland

A photorefractive grating in a slab waveguide based on a semi-insulating AlGaAs/GaAs multiple quantum well structure with an electric field applied along the quantum well planes as an optically controlled, frequency selective mode coupling element with memory is analysed.

PACS numbers: 42.65.-k, 42.65.Pc

\section{Introduction}

Diffraction gratings are commonly used in integrated optics systems as reflectors, couplers, and frequency filters. Traditional waveguide gratings have constant parameters and are sensitive for any production imperfections. Optically induced gratings are adjustable and therefore more independent on fabrication errors and on the influence of external conditions. The grating created by two mutually coherent waves interfering in a nonlinear material can be used for the same purposes as stable grating but the obtained elements can be tuned in real time by varying parameters of the waves [1]. The grating created in photorefractive material does not require permanent illumination. It can be created by a short writing pulse and last until the arrival of the next pulse which can renew or erase it $[2,3]$.

The grating analyzed here is induced in a single-mode planar waveguide containing semi-insulating multiple quantum well (MQW) layer. The MQW structure

${ }^{*}$ corresponding author; e-mail: ewa.raczka@ps.pl 
operates in so-called transverse Franz-Keldysh geometry with the external electric field applied along the quantum well planes $[4,5]$ as it is shown in Fig. 1.

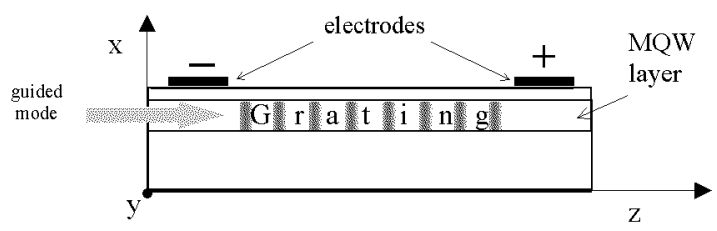

Fig. 1. Photorefractive waveguide geometry.

The grating can be created by two guided modes or by two external beams. The interference between the beams overlapping in the MQW layer gives the intensity pattern described by

$$
I(x, y, z)=I_{0}(x, y, z)[1+m \cos (K z)]
$$

where $I_{0}(x, y, z)$ is a slowly varying function describing transverse intensity distribution of the beams and $m$ is the modulation depth (fringes visibility). The grating constant $K$ depends on the incidence angles of the interfering beams.

A high frequency light creates electron-hole pairs by direct interband transitions. The carriers are excited in bright regions of the interference pattern and subsequently move due to the drift and diffusion. Finally the carriers recombine to the donor traps mainly in the dark regions, creating a spatially modulated charge distribution. A non-uniform charge distribution builds up a space-charge electric field. The space-charge electric field changes the refractive index through the electro-optic effect. An external electric field assists charge separation through the drift and increase the space-charge field. The influence of the external field is specially important in the case of an ambipolar diffusion when the results of electron and hole diffusion currents substrate. The refractive index grating can influence signals propagating in the waveguide leading to selective reflection, deflection, out-coupling or change of the polarisation state [6-14]. If the operated signals have frequency below the absorption threshold their influence on the grating can be neglected.

\section{Charge transport model}

The transport equations that describe photorefractive effect in semi-insulating MQW in assumed geometry are similar to equations that describe the photorefractive effect in bulk semiconductors [3]. The impurity levels in the structure are described by one deep-level of donor traps and one shallow-level of acceptors which compensate donors. Moreover it is assumed that photogenerated carriers are confined to the quantum wells and therefore the photorefractive transport properties may be described by a set of one-dimensional equations 


$$
\begin{aligned}
& \frac{\partial n_{\mathrm{e}}}{\partial t}=\frac{\alpha}{h \nu} I-\gamma_{\mathrm{e}} n_{\mathrm{e}} N_{\mathrm{D}}^{+}+\frac{1}{e} \frac{\partial j_{\mathrm{e}}}{\partial z}+\beta_{\mathrm{e}}\left(N_{\mathrm{D}}-N_{\mathrm{D}}^{+}\right)-\gamma_{\mathrm{eh}} n_{\mathrm{e}} n_{\mathrm{h}}, \\
& \frac{\partial n_{\mathrm{h}}}{\partial t}=\frac{\alpha}{h \nu} I-\gamma_{\mathrm{h}} n_{\mathrm{h}}\left(N_{\mathrm{D}}-N_{\mathrm{D}}^{+}\right)-\frac{1}{e} \frac{\partial j_{\mathrm{h}}}{\partial z}+\beta_{\mathrm{h}} N_{\mathrm{D}}^{+}-\gamma_{\mathrm{e}} \mathrm{h} n_{\mathrm{e}} n_{\mathrm{h}} \\
& j_{\mathrm{e}}=e \mu_{\mathrm{e}} n_{\mathrm{e}} E+\mu_{\mathrm{e}} k_{\mathrm{B}} T \frac{\partial n_{\mathrm{e}}}{\partial z} \\
& j_{\mathrm{h}}=e \mu_{\mathrm{h}} n_{\mathrm{h}} E+\mu_{\mathrm{h}} k_{\mathrm{B}} T \frac{\partial n_{\mathrm{h}}}{\partial z} \\
& \frac{\partial N_{\mathrm{D}}^{+}}{\partial t}=\left(\gamma_{\mathrm{h}} n_{\mathrm{h}}+\beta_{\mathrm{e}}\right)\left(N_{\mathrm{D}}-N_{\mathrm{D}}^{+}\right)-\left(\gamma_{\mathrm{e}} n_{\mathrm{e}}+\beta_{\mathrm{h}}\right) N_{\mathrm{D}}^{+} \\
& \frac{\partial E}{\partial z}=\frac{e}{\varepsilon \varepsilon_{0}}\left(N_{\mathrm{D}}^{+}+n_{\mathrm{h}}-n_{\mathrm{e}}-N_{\mathrm{A}}\right),
\end{aligned}
$$

where subscripts e and h mark electron and hole properties, respectively, $n$ denotes the free carriers concentration, $N_{\mathrm{D}}$ - donors (traps), $N_{\mathrm{D}}^{+}$- ionised donors and $N_{\mathrm{A}}$ - acceptors concentrations, $j$ - the current density, $E$ - the total electric field ( $E=E_{\mathrm{a}}+E_{\mathrm{s}} \mathrm{c}$, where $E_{\mathrm{a}}$ is an external field and $E_{\mathrm{sc}}$ is a space charge field), $I$ - the light intensity, $\alpha$ - the absorption coefficient, $h \nu$ - the photon energy of writing beams, $\beta$ - the thermal excitation rate, $\gamma$ - the trapping coefficient describing recombination of carriers to the donors traps, $\gamma_{\mathrm{eh}}$ - the direct recombination coefficient, $\mu$ - carrier mobility along the quantum wells, $\varepsilon_{0}$ - the permittivity of the vacuum, $\varepsilon$ - the effective dielectric constant of MQW structure, $e$ - the absolute value of the elementary charge, $k_{\mathrm{B}}$ - the Boltzmann constant and $T$ - the absolute temperature.

In the following calculations the thermal excitation rate which is much smaller than the photo generation rate, and direct recombination rate which is much smaller than the ion recombination rate are neglected. Transport nonlinearity due to the carrier mobilities dependence on applied electric field is also not considered.

Under the steady-state conditions and for light intensity given by (1) all variables in Eqs. (2-7) can be presented in a form of Fourier series [15]

$$
\left[\begin{array}{c}
n_{\mathrm{e}} \\
n_{\mathrm{h}} \\
N_{\mathrm{D}} \\
j_{\mathrm{e}} \\
j_{\mathrm{h}} \\
E
\end{array}\right]=\left[\begin{array}{c}
n_{\mathrm{e} 0} \\
n_{\mathrm{h} 0} \\
N_{0}^{+} \\
j_{\mathrm{e} 0} \\
j_{\mathrm{h} 0} \\
E_{\mathrm{a}}
\end{array}\right]+\left[\begin{array}{c}
n_{\mathrm{e} 1} \\
n_{\mathrm{h} 1} \\
N_{1}^{+} \\
j_{\mathrm{e} 1} \\
j_{\mathrm{h} 1} \\
E_{1 m}
\end{array}\right] \exp (\mathrm{i} K z)+\left[\begin{array}{c}
n_{\mathrm{e} 2} \\
n_{\mathrm{h} 2} \\
N_{2}^{+} \\
j_{\mathrm{e} 2} \\
j_{\mathrm{h} 2} \\
E_{2 m}
\end{array}\right] \exp (\mathrm{i} 2 K z)+
$$




$$
+\left[\begin{array}{c}
n_{\mathrm{e} 3} \\
n_{\mathrm{h} 3} \\
N_{3}^{+} \\
j_{\mathrm{e} 3} \\
j_{\mathrm{h} 3} \\
E_{3 m}
\end{array}\right] \exp (\mathrm{i} 3 K z)+\ldots
$$

As a result an electric field can be presented as:

$$
\begin{gathered}
E(z)=E_{\mathrm{a}}+m E_{1} \exp (\mathrm{i} K z)+m^{2} E_{2} \exp (\mathrm{i} 2 K z) \\
+m^{3} E_{3} \exp (\mathrm{i} 3 K z)+\ldots,
\end{gathered}
$$

where $E_{i}=E_{i m} / m^{i}$ are generally complex numbers due to the arbitrary phase shift in respect to the interference pattern. The zero-order solutions for carrier concentrations

$$
n_{\mathrm{e} 0}=\frac{(\alpha / h \nu) I_{0}}{\gamma_{\mathrm{e}} N_{0}^{+}}, \quad n_{\mathrm{h} 0}=\frac{(\alpha / h \nu) I_{0}}{\gamma_{\mathrm{h}}\left(N_{\mathrm{D}}-N_{0}^{+}\right)}
$$

show that for moderate illumination (up to $10 \mathrm{~W} / \mathrm{cm}^{2}$ ) the free carriers density is much smaller than the ionised donors density. Under this assumption the first Fourier component of the electric field can be described as

$$
E_{1}=(A-\mathrm{i} B) /(C+\mathrm{i} D),
$$

where

$$
\begin{aligned}
& A=E_{d}\left(E_{\mu \mathrm{h}}-E_{\mu \mathrm{e}}\right), \quad B=E_{\mathrm{a}}\left(E_{\mu \mathrm{h}}+E_{\mu \mathrm{e}}\right), \quad C=E_{\mathrm{a}}\left(E_{\mu \mathrm{h}} / E_{q 1}-E_{\mu \mathrm{e}} / E_{q 2}\right), \\
& D=\left(E_{\mu \mathrm{h}}+E_{\mu \mathrm{e}}\right)+E_{d}\left[E_{\mu \mathrm{e}} / E_{q 2}+E_{\mu \mathrm{h}} / E_{q 1}+2\right]+\left(1 / E_{q 2}+1 / E_{q 1}\right)\left(E_{d}^{2}+E_{\mathrm{a}}^{2}\right)
\end{aligned}
$$

and characteristic fields

$$
\begin{aligned}
& E_{q 1}=r N_{\mathrm{D}} e /(\varepsilon K), \quad E_{q 2}=(1-r) N_{\mathrm{D}} e /(\varepsilon K), \\
& E_{\mu \mathrm{e}}=r N_{\mathrm{D}} \gamma_{\mathrm{e}} /\left(K \mu_{\mathrm{e}}\right), \quad E_{\mu \mathrm{h}}=(1-r) N_{\mathrm{D}} \gamma_{\mathrm{h}} /\left(K \mu_{\mathrm{h}}\right), \\
& E_{d}=k_{\mathrm{B}} T K / e, \quad r=N_{\mathrm{A}} / N_{\mathrm{D}} .
\end{aligned}
$$

The symbols used in the solutions are the same as in [15] and the results obtained for $r=0.5$ and $\gamma_{e}=\gamma_{\mathrm{h}}=\gamma$ are analogous. The amplitudes of higher harmonics, $E_{2}, E_{3}$, etc. have more complicated analytic form and only the results of numerical solutions obtained for the parameters listed in Table I are presented. The dependence of the amplitudes and phase shifts of harmonics components on the grating constant for $E_{\mathrm{a}}=10^{4} \mathrm{~V} / \mathrm{cm}$ and $N_{\mathrm{D}}=2 \times 10^{18} \mathrm{~cm}^{-3}$ is drawn in Fig. 2 and Fig. 3. The results presented in Fig. 2 show that the higher order harmonics are comparable with the first order one only for small grating constants. For the grating constant exceeding $1 \times 10^{5} \mathrm{~cm}^{-1}$ the higher order amplitudes can be neglected even in the case of $m \approx 1$. For the small value of the modulation depth $(m \ll 1)$ contribution of $n$-th order component, which is multiplied by $m^{n}$, decrease and is negligible even in the case of a small grating constant. 
TABLE I

Parameters used in calculations.

\begin{tabular}{l|l}
\hline \hline$\mu_{\mathrm{e}}=5000 \mathrm{~cm}^{2} /(\mathrm{V} \mathrm{s})$ & the electron mobility* \\
$\mu_{\mathrm{h}}=300 \mathrm{~cm}^{2} /(\mathrm{V} \mathrm{s})$ & the hole mobility* \\
$\gamma_{\mathrm{e}}=\gamma_{\mathrm{h}}=10^{-7} \mathrm{~cm}^{3} / \mathrm{s}$ & the trapping coefficient* \\
$m \cong 1$ & the fringes visibility \\
$\varepsilon=n_{f}^{2}=12.586$ & the effective dielectric constant of MQW layer \\
$r=N_{\mathrm{A}} / N_{\mathrm{D}}=0.5$ & the compensation ratio of donors traps \\
\hline
\end{tabular}

${ }^{*}$ Values of carrier mobilities and trapping coefficient were derived from [15].

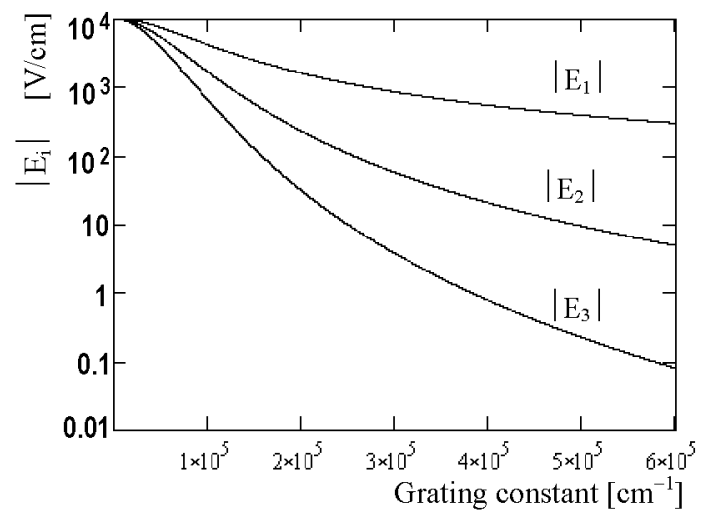

Fig. 2. First three harmonic components of the space charge field versus the grating constant for $E_{\mathrm{a}}=10^{4} \mathrm{~V} / \mathrm{cm}$ and $N_{\mathrm{D}}=2 \times 10^{18} \mathrm{~cm}^{-3}$.

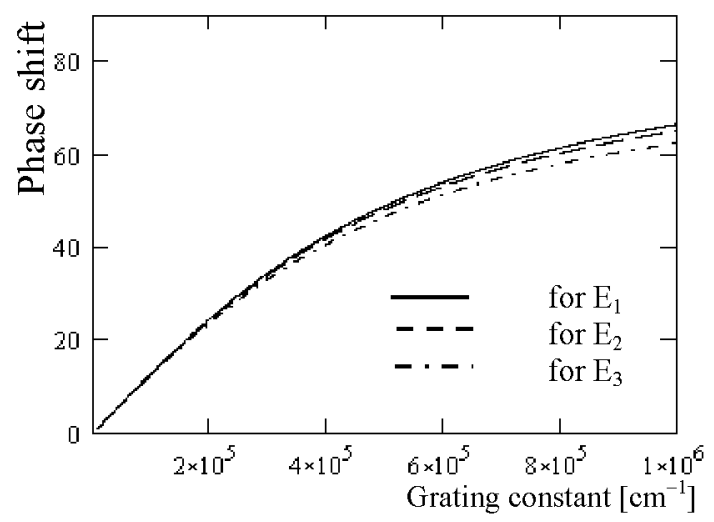

Fig. 3. Phase shifts of the space charge field harmonic components versus the grating constant for $E_{\mathrm{a}}=10 \mathrm{kV} / \mathrm{cm}$ and $N_{\mathrm{D}}=2 \times 10^{18} \mathrm{~cm}^{-3}$. 


\section{Absorption and electrorefraction spectra}

The influence of the electric field on the optical properties of MQW is mainly due to the change of the exciton absorption. The absorption spectrum contains two peaks corresponding to creation of heavy hole ( $\mathrm{HH}$ ) and light hole ( $\mathrm{LH}$ ) excitons and a continuous contribution from the absorption to free electron - hole pairs. In the presence of the electric field the peaks broaden and decrease due to the ionisation of excitons. The spectrum near the absorption edge can be approximated by the following expression [16]:

$$
\begin{aligned}
\alpha(\lambda) & =\alpha_{\mathrm{h}} \exp \left[-\frac{\left(\lambda-\Lambda_{\mathrm{h}}\right)^{2}}{2 \Gamma_{\mathrm{h}}^{2}}\right]+\alpha_{1} \exp \left[-\frac{\left(\lambda-\Lambda_{\mathrm{l}}\right)^{2}}{2 \Gamma_{\mathrm{l}}^{2}}\right] \\
& +\frac{\alpha_{\mathrm{c}}}{1+\exp \left[\left(\lambda-\Lambda_{\mathrm{c}}\right) / \Gamma_{\mathrm{c}}\right]},
\end{aligned}
$$

where $\alpha_{i}, \Lambda_{i}, \Gamma_{i}$ denote height, position, and width of absorption Gaussian peaks, $i=\mathrm{h}$ for heavy holes, and $i=1$ for light holes, whereas $\alpha_{\mathrm{c}}, \Lambda_{\mathrm{c}}, \Gamma_{\mathrm{c}}$ denote parameters of continuous contribution. The approximated absorption spectra for $\mathrm{GaAs} / \mathrm{Al}_{0.3} \mathrm{Ga}_{0.7} \mathrm{As}$ multiple quantum well structure at room temperature are shown Fig. 4. A dashed line presents the results obtained for the sample placed in the external electric field of $10 \mathrm{kV} / \mathrm{cm}$ and a solid line - for the sample without the field. The parameters used in the calculations which are listed in Table II were based on the data quoted in paper [17].

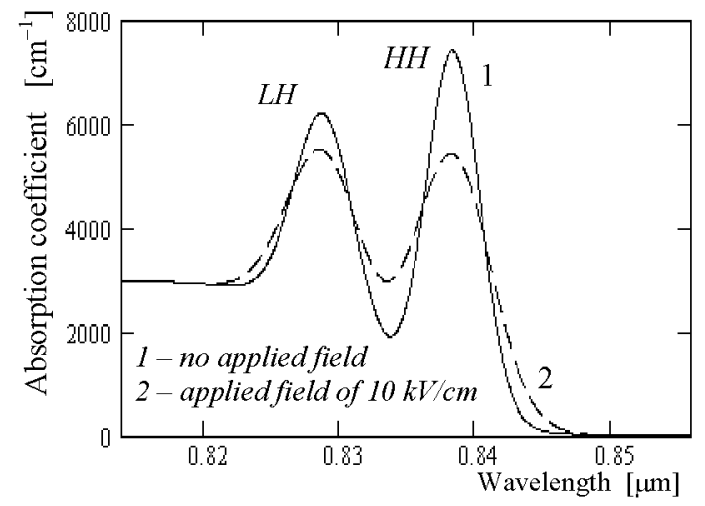

Fig. 4. Absorption spectra of MQW approximated by a sum of two Gaussian peaks and a continuous contribution obtained for a sample with and without an applied electric field.

The change of the refractive index caused by the electric field can be calculated from Kramers-Krönig relation between $\Delta n$ and $\Delta \alpha$ spectra $[4,18]$

$$
\Delta n(\lambda)=\frac{\lambda^{2}}{2 \pi^{2}} P \int_{0}^{\infty} \frac{\Delta \alpha\left(\lambda^{\prime}\right) \mathrm{d} \lambda^{\prime}}{\lambda^{2}-\lambda^{\prime 2}}
$$


TABLE II

Parameters of the absorption spectrum approximation.

\begin{tabular}{l|c|c|c|c|c}
\hline \hline Contribution & $\alpha\left[\mathrm{cm}^{-1}\right]$ & $\Lambda[\mu \mathrm{m}]$ & $\Gamma[\mathrm{nm}]$ & $\Delta \alpha\left[\mathrm{cm}^{-1}\right]$ & $\Delta \Gamma[\mathrm{nm}]$ \\
\hline Heavy hole & 7000 & 0.838 & 1.985 & -2000 & 0.794 \\
Light hole & 4000 & 0.829 & 2.162 & -720 & 0.499 \\
Continuum & 3000 & 0.833 & 3.353 & 0 & 0
\end{tabular}

where $\Delta \alpha=\alpha(E)-\alpha(0)$ and $P$ means the principal value of the integral. The dependence of the refractive index change on the wavelength calculated from (11) is presented in Fig. 5. The absorption and electrorefraction spectra define the wavelengths of interacting beams. The guided modes reading the grating should have the wavelength about $850 \mathrm{~nm}$ which is a compromise between relatively low absorption and possibly high electrorefraction. Guided modes writing the grating should have a slightly shorter wavelength to enable sufficient photogeneration of the carriers.

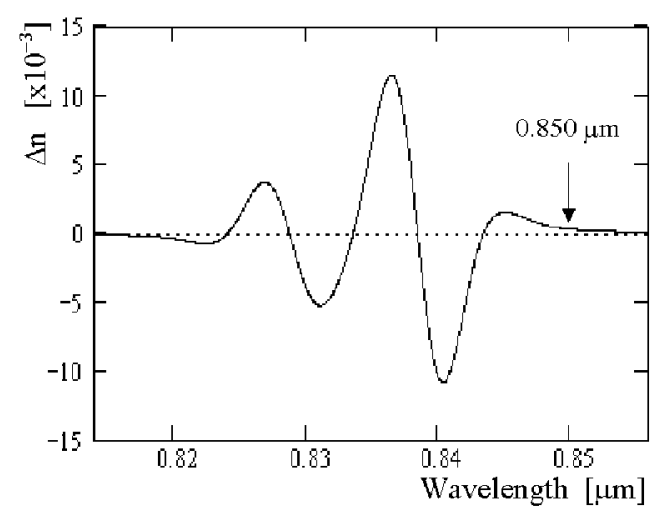

Fig. 5. Changes of the refractive index near the fundamental absorption edge for an applied electric field of $10 \mathrm{kV} / \mathrm{cm}$.

In the case of the grating created by the external waves the way of the beams through the active layer is very short and much higher frequency (corresponding to the wavelength of about $630 \mathrm{~nm}$ ) which enables generation of carriers in both wells and barriers is possible. The space-charge field obtained this way is more robust to the influence of the carriers generated by the waves interacting with the grating [19]. Figure 6 shows the amplitude of the refractive index grating as a function of the grating space period. The obtained values of amplitude are high enough to provide interactions between the modes guided in the structure [14, 20]. Gratings with different space periods can be used for different purposes like frequency selective reflection, deflection or out-coupling of modes guided in the structure. The possible application of the grating as a Bragg reflector is analysed in [21] 


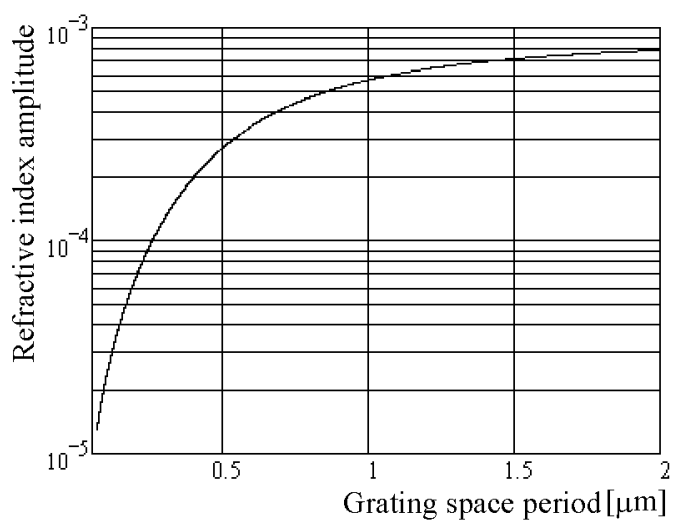

Fig. 6. Refractive index grating amplitude in the steady state in dependence on the grating space period for the applied field $E_{\mathrm{a}}=10 \mathrm{kV} / \mathrm{cm}$ and the signal mode wavelength $\lambda_{G}=850 \mathrm{~nm}$.

and properties of the photorefractive grating assisted directional coupler are presented in [2].

\section{Conclusions}

The results of calculations show that a photorefractive grating in semi-insulating MQW single mode waveguide operating with the electric field applied along the QW planes can be used as an optically controlled frequency selective mode coupler with memory. The grating parameters can be tuned during the work of the device. The grating does not require a permanent presence of the external waves. Pump pulses are necessary only for writing, refreshing or erasing of the grating. Because signal waves with low frequency do not destroy the grating the system may have an application as an all-optical switching element with memory.

\section{References}

[1] E. Weinert-Rączka, J. Opt. Soc. Am. B 11, 2340 (1994).

[2] E. Weinert-Rączka, R. Iwanow, Acta Phys. Pol. A 99, 175 (2001).

[3] J.P. Huignard, P. Günter, Photorefractive Materials and their Applications I, Springer-Verlag, Berlin 1988.

[4] A.M. Glass, D.D. Nolte, H.D. Olson, G.E. Doran, D.S. Chemla, W.H. Knox, Opt. Lett. 15, 264 (1990).

[5] D.D. Nolte, D.H. Olson, G.E. Doran, W.H. Knox, A.M. Glass, J. Opt. Soc. Am. $B$ 7, 2217 (1990).

[6] A. Yariv, M. Nakamura, IEEE J. Quantum Electron. 13, 233 (1977).

[7] D.G. Hall, Prog. Opt. 29, 1 (1991). 
[8] P. Yeh, A. Yariv, Opt. Commun. 19, 427 (1976).

[9] C. Elachi, P. Yeh, Opt. Commun. 7, 201 (1973).

[10] P. Yeh, H.F. Taylor, Appl. Opt. 19, 2848 (1980).

[11] D. Marcuse, J. Lightwave Technol. LT-5, 268 (1987).

[12] H. Kogelnik, C.V. Shank, J. Appl. Phys. 43, 2327 (1972).

[13] S.S. Orlov, A. Yariv, S.V. Essen, Opt. Lett. 22, 688 (1997).

[14] H. Kogelnik, Theory of Optical Waveguides, in: Guided Wave Optoelectronics, Ed. T. Tamir, Springer-Verlag, Berlin 1990, p. 74.

[15] L.F. Magaña, F. Agulló-López, M. Carrascosa, J. Opt. Soc. Am. B 11, 1651 (1994).

[16] D.S. Chemla, D.A.B. Miller, P.W. Smith, A.C. Gossard, W. Wiegmann, IEEE J. Quantum. Electron. 20, 265 (1984).

[17] Q. Wang, R.M. Brubaker, D.D. Nolte, M.R. Melloch, J. Opt. Soc. Am. B 9, 1626 (1992).

[18] D.C. Hutchings, M. Sheik-Bahae, D.J. Hagan, E.W. Van Stryland, Opt. Quantum Electron. 24, 1 (1992).

[19] D.D. Nolte, Q. Wang, Appl. Phys. Lett. 58, 2067 (1991).

[20] J. Petykiewicz, Podstawy fizyczne optyki scalonej, PWN, Warszawa 1989 (in Polish).

[21] M. Wichtowski, E. Weinert-Rączka, Acta Phys. Pol. A 103, (2003). 